\title{
Gerencia para transformar las universidades públicas autónomas de Venezuela
}

\author{
Romero M., Néstor L.*
}

\section{Resumen}

Existe en Venezuela la urgente necesidad de transformar nuestra educación en eficaz y eficiente instrumento de desarrollo personal y colectivo, tal como la define nuestra Constitución Nacional aprobada en 1.999. Las Universidades Públicas Autónomas por sus funciones y por su ubicación en la cúspide del sistema educativo, están obligadas a asumir esa nueva misión. Aunque la transformación debe ser integral, abarcar todas las funciones y servicios que prestan las universidades, nos limitaremos en este trabajo sólo a la gerencia, por ser en estos niveles en donde se deben tomar las decisiones de planificar, dirigir, evaluary controlar esos procesos de cambio. El objetivo es hacer un análisis crítico de algunos aportes de la Teoría Gerencial que consideramos aplicables y del modelo de universidad contenido en nuestra vigente Ley de Universidades a fin de contrastar esas teorías con el modelo y detectar algunos problemas que, hasta ahora, han impedido que anteriores intentos de transformación se hicieran realidad. Partiendo de la complejidad que caracteriza a estas organizaciones, se aplicó el método de análisis crítico y hermenéutico para obtener algunas conclusiones que nos permitieron hacer una recomendación.

Palabras clave: Gerencia de la transformación universitaria, reforma universitaria, gerencia de la educación superior, gerencia del cambio, universidades públicas autónomas.

\section{Management to Transform the Public Autonomous Universities in Venezuela}

\section{Abstract}

In Venezuela, there is an urgent need for transforming the educational system into an effective, efficient instrument for personal and collective development, as defined in the 1999 National Constitution. Public Autonomous Universities are obliged to assume this new mission due to their functions and position at the top of the educational system. Even though the transformation should be integral and involve the whole spectrum of functions and services performed by the universities, the present study will be limited only to university management, since it is on these levels that decisions must be

Recibido: 24-05-07. Aceptado: 21-07-07

Economista, Profesor Titular, Universidad del Zulia (LUZ). Cursante del Doctorado en Ciencias Sociales (LUZ). Maracaibo, Venezuela. E-mail: nromerom@cantv.net 
taken for planning, execution, evaluation and control of those change processes. The objective of this study is to perform a critical analysis of some contributions from management theory deemed applicable and from the university model set forth in the current Law of Universities, in order to contrast these theories with the model and detect any problems which have prevented previous transformation attempts from becoming reality. Due to the complexity characterizing these organizations, analytical, critical and hermeneutic methods were applied to obtain conclusions that would allow recommendations to be made.

Key words: Management of university transformation, university reform, management of higher education, management for change, public autonomous universities.

\section{Introducción}

Las Universidades Autónomas, pioneras de la Educación Superior de Venezuela, han cumplido un extraordinario papel en la formación de personal académico y profesional del más alto nivel que ha hecho posible los más importantes avances de todo nuestro sistema educativo, científico y tecnológico. En consecuencia, nuestro nivel de desarrollo actual es, en gran medida, resultado de la acción de esas Instituciones. Sin embargo, a pesar de esos logros, aún continúan presente una multiplicidad de complejos problemas, amenazas, vicios y dificultades de diferente naturaleza y atribuibles a diferentes causas, que es necesario analizar y superar para que puedan continuar en el cumplimiento de su misión con el nivel de eficacia, calidad, eficiencia y pertinencia social que en estos momentos nuestra sociedad espera de ellas.

En la última década del siglo XX algunos organismos financieros internacionales con el pretexto de ayudar a superar la crisis de 1.980 en América Latina, basándose en el "Consenso de Washington" "recomendaron" la aplicación de la "Política de Ajuste Estructural" también denominada aquí en Venezuela "paquete neoliberal" que entre otras medidas exi- gía la reducción del Estado, la privatización de servicios públicos (educación, salud, etc.) y la reducción de esos mismos "gastos" (Yarzábal, 1999), desde entonces hasta ahora, en Venezuela algunos sectores públicos, académicos y privados continúan intentando imponer a las Universidades Autónomas, los criterios economicistas de eficiencia, productividad, calidad, relación beneficio-costo propios del sistema capitalista neoliberal.

Tales criterios sólo le son aplicables a las Universidades si las concebimos como una fábrica y a la investigación como un proceso de producción de mercancías y servicios en función del mercado y de la ganancia dentro de la racionalidad del sistema capitalista neoliberal (Ibarra, 1993).

Si además tomamos en cuenta que la Constitución de la República Bolivariana de Venezuela, aprobada en 1.999 contiene un nuevo proyecto de país, en el cual se le asigna a la educación la misión de ser instrumento del desarrollo personal y colectivo (Asamblea Nacional Constituyente 1999: artículo 3) se hace indispensable y urgente que las mismas Universidades Autónomas, en ejercicio de esa autonomía, realicen también un proceso de transformación que les permita asumir esa nueva misión y demostrar con 
hechos incuestionables su capacidad de análisis y de respuestas científicas a los nuevos retos que le ha planteado ese entorno.

Aun cuando las Universidades Públicas Autónomas Venezolanas en diferentes oportunidades han anunciados procesos de transformación y se han hecho propuestas, algunas durante procesos de elección de sus respectivas autoridades, la Asociación Venezolana de Rectores Universitarios (AVERU) en el documento "Estrategias de cambio para la Educación Superior Venezolana" (2001) reconoce la permanencia de la urgente necesidad de cambio y al efecto, estableció las bases conceptuales, doctrinarias y principios para llevar a cabo un proceso de transformación que debía ser asumido por cada una de las Instituciones participantes.

Aplicando el análisis crítico y hermenéutico como un proceso interpretativo que va del todo a las partes y de las partes al todo en el contexto del conjunto dinámico de elementos interrelacionados (Muro, 2004) tanto a las teorías gerenciales como al modelo de organización y gerencia que contiene nuestra Ley de Universidades, obtuvimos como resultado algunas conclusiones que nos sirvieron de base para hacer una recomendación que esperamos pueda contribuir a mejorar los niveles de eficacia, calidad, eficiencia y pertinencia social de las Universidades Públicas Autónomas de Venezuela.

\section{Algunas precisiones necesarias}

Tanto Frederick Taylor en "Principios de la Administración Científica" (1983) como Henry Fayol en "Administra- ción Industrial y General" (1983) a quienes se les considera los "padres" de la Administración Científica, ven a la Administración como una ciencia con sus propios métodos, principios y reglas o leyes, y a la Gerencia la tratan como una de las funciones de la Administración: la Dirección. En la descripción que hace Fayol de las operaciones que se desarrollan en las empresas, las clasifica en los 6 grupos siguientes: Operaciones Técnicas, Comerciales, Financieras, de Seguridad, de Contabilidad y Administrativas y dentro de estas últimas considera: la Previsión, Organización, Dirección, Coordinación y Control.

En la época actual observamos la tendencia a ver a la gerencia como lo más general y a la administración como una de las funciones de la gerencia. Esto implica darle más importancia a las funciones de Previsión (planificación), Dirección, Coordinación, Control y Evaluación que a su vez están más vinculadas con el ejercicio del poder y la toma de las decisiones más importantes, que con los procedimientos y las tareas necesarias para la ejecución de esas decisiones.

En este trabajo utilizaremos el término Gerencia en este sentido amplio, compartiendo así la concepción de Drucker (1992), según la cual el gerente no es un jefe, un manager, porque no necesariamente tiene que tener personal bajo su coordinación y supervisión, sino que tiene como función planificar, dirigir, controlar y evaluar el desempeño y el logro de los objetivos y metas de una empresa privada o pública. Son entonces las funciones y su importancia dentro de las organizaciones, los elementos utilizados para definir a la gerencia como término 
más apropiado que el de administración o el de "teoría de la Organización" (TO) utilizado por Ibarra en su análisis sobre la educación superior (Ibarra (1996).

Si embargo hay que aclarar que cada organización humana tiene objetivos y características propias que le dan su especificidad organizacional y factores contextuales que inciden en su estructura (Ibarra, 1996), pero también hay principios de la economía y teorías gerenciales que son lo suficientemente generales que pueden ser aplicables no sólo a las empresas privadas, sino también a toda organización humana.

Lógicamente el desempeño y el logro de objetivos y metas, implica la aplicación del "Principio Económico Fundamental" que Ochoa (1995) denomina "el principio de la racionalidad económica" que sirve para entender el funcionamiento del proceso productivo. Según este principio en todo proceso productivo se plantea el problema de que existen múltiples necesidades ilimitadas y recursos limitados que tienen usos alternativos, por lo que resulta indispensable hacer un uso, lo más racional posible, de esos limitados recursos para poder obtener la mayor satisfacción posible de las diversas necesidades.

Consideramos necesario hacer esta precisión porque sobre este principio se han hecho exageradas aplicaciones y se ha generado la falsa creencia de que no se debe aplicar en el sector público. Aun cuando los fines del Estado y de sus Instituciones fundamentales no son obtener el máximo beneficio monetario al menor costo, la racionalidad del mencionado principio exige evitar o minimizar el desperdicio, la contaminación, la ociosidad, las deficiencias y al mismo tiempo producir el mejor servicio en términos de eficacia, calidad, eficiencia, rapidez, población servida, etc.

El argumento de que la eficiencia, la maximización del beneficio y la reducción de los costos genera desempleo de la mano de obra, puede ser manejado dándole el "uso alternativo" que caracteriza a los recursos, es decir reubicando al personal que esté subutilizado, en otras actividades en las cuales pueda generar beneficios adicionales a la población a ser servida. Taylor (1983) demuestra que la aplicación del principio de la racionalidad económica no es perjudicial al trabajador y por el contrario es necesario para el progreso de toda la economía y la generación de puestos de trabajo.

La complejidad de "La Gran Empresa", originada a su vez por la denominada Revolución Científico-Técnica, que llegó a convertirse a mediados del siglo pasado en el tipo de organización a través de la cual se dirige la mayor parte de la producción (Ochoa, 1995), produjo cambios en la dirección y en el propio sujeto de dirección que pasó a ser un ente colectivo (Asamblea de Accionistas).

Los avances en la electrónica, la computación, las comunicaciones y el manejo de la información, han constituido nuevos y poderosos instrumentos que han aumentado el poder de la "gran empresa" y generado nuevos cambios en la división del trabajo y en su organización. También se produjeron cambios en los controles y en la evaluación por parte de los propietarios del capital sobre los gerentes, aplicando las técnicas de planificación estratégica, el control de gestión y las auditorías externas. 
Aunque todavía sobreviven algunas empresas pequeñas cuya dirección la ejercen los mismos propietarios, se ha generalizado hasta los organismos públicos el uso de entes colectivos y la incorporación de Tecnócratas para gerenciar las Instituciones Públicas, dando así participación a profesionales, especialistas y técnicos que aporten sus conocimientos y, al mismo tiempo, se estimula así, su identificación con la Institución.

En el caso de las Universidades Públicas Autónomas corresponde a sus respectivas gerencias colectivas (Consejo Universitario, Consejo de Facultad, etc.) en forma general tomar las decisiones más importantes y de manera especial, promover, diseñar, controlar y evaluar, entre otros, los programas de transformación que cada Institución considere necesario dentro del marco que le señala la respectiva Ley de Universidades y de acuerdo a las características de cada Institución.

Por tanto, aun cuando la transformación debe ser integral, abarcar a la totalidad de la Institución y de las actividades que realiza, el proceso debe comenzar con el análisis de los organismos de decisión puesto que son ellos los que tienen la responsabilidad de diseñar y promover el programa de transformación y las estrategias para su puesta en marcha, el control y la evaluación.

\section{El modelo universitario vigente en Venezuela}

En la ley de universidades de Venezuela vigente desde el 08/09/1970 se define a la universidad como "una comunidad de intereses espirituales que reúne a profesores y estudiantes en la tarea de buscar la verdad y afianzar los valores trascendentales del hombre" (Congreso de la República de Venezuela 1970: art. 1).

Esa definición parte del supuesto de que profesores y estudiantes forman una comunidad de intereses espirituales que, aunque cada uno de los individuos que conforman esa comunidad tenga otros intereses diferentes, todos tienen un fin común: buscar la verdad y afianzar los valores trascendentales del hombre. Ese fin, si es compartido por todos y cada uno de los integrantes de esa comunidad, los une y se constituye en lo que en derecho se conoce como: el ánimo de asociación, que es uno de los requisitos indispensables para que se considere que existe una sociedad o persona jurídica distinta a cada uno de sus integrantes.

Sin embargo existen fundados indicios que nos obligan a dudar que se haya logrado en las universidades venezolanas conformar una verdadera comunidad cuyo objetivo común y unificador sea la búsqueda de la verdad. Será necesario hacer un diagnóstico para determinar el grado de cohesión alcanzado en cada institución y diseñar las estrategias correspondientes para que todos y cada uno de los integrantes de esa comunidad (profesores y estudiantes) internalicen y se identifiquen con la noble misión colectiva de buscar la verdad y afianzar los valores trascendentales del hombre, u otra misión que se proponga en un nuevo modelo de universidad.

Lograr la identificación de los integrantes de cada comunidad universitaria con los objetivos y metas de la Institución constituye uno de los mecanismos de control más eficaces para lograr el éxito en su misión. 
La expresión "buscar la verdad", como dice Nicol (1965: 68) es más bien una manifestación de la vocación científica que deben tener los miembros integrantes de la comunidad universitaria por encima de cualquier otro interés. Pero hoy a la luz de los aportes de nuevas teorías debemos establecer algunas precisiones: 1) Parece que se busca la verdad porque no se ha encontrado ni se encuentra, y esto oculta el hecho de que el proceso de conocer siempre parte de un nivel de verdad hacia otros niveles cada vez más precisos y cercanos a la verdad científica alcanzada en determinado momento histórico. 2) En la actualidad hay la tendencia a considerar que no existe verdad absoluta, sino que se trata de un proceso de permanente búsqueda de cada vez mayores niveles de precisión en el conocimiento del hombre, la sociedad y de todo el universo en general.

Afianzar los valores trascendentales del hombre, constituye la segunda tarea fundamental de las universidades venezolanas. Sin embargo no está claro cuáles son esos valores. ¿Serán los que algunos organismos internacionales denominan derechos humanos? ¿Podrán las universidades proponer otros valores? Al usarel legislador la palabra "afianzar" ¿Se podrá interpretar que el tema de "Ios valores trascendentales del hombre no puede ser analizado críticamente por las universidades, sino que se dan por inmutables? La creación y desarrollo del conocimiento científico no es posible si se limita la libertad para investigar. El nuevo modelo que se proponga debe definir los valores y principios en que se sustenta y dejar abierta la posibilidad de revisión y desarrollo.
Respecto a la libertad, las universidades disponen de autonomía organizativa, académica, administrativa y económica que las habilita para investigar libremente cualquier área del conocimiento o tema, pero como es lógico no son instituciones aisladas, sino que forman parte de un sistema mayor y más complejo definido en nuestra constitución (tanto la anterior como la vigente) y en consecuencia esa "parte" debe estar supeditada a el "todo" que es el Estado. ¿Se justifica entonces que las universidades estén subordinadas y coordinadas por el Estado y que todas sus actividades estén al servicio de la nación?

La subordinación y coordinación de las universidades por el Estado, así como su misión están claramente establecidas en la respectiva ley y no hay lugar a dudas de que la intención del legislador fue la de asignarle la misión de asimilar, crear, desarrollar, difundir y aplicar el conocimiento y la educación al servicio del desarrollo y el progreso de la nación. No al servicio del sistema, ni del gobierno, ni de determinada ideología, ni del mercado, ni de las empresas privadas nacionales, ni mucho menos las trasnacionales. Tampoco se debe caer en el extremo, como ha ocurrido en Venezuela, en que algunos miembros de la comunidad de algunas universidades han tratado de convertirlas en apéndices de partidos políticos opositores al gobierno que esté de turno o en general opositores al sistema.

El problema de fondo que está involucrado es si la asimilación, creación, desarrollo y acumulación del conocimiento científico, es decir, la ciencia, nos libera, nos desarrolla o nos esclaviza y destruye. 
Al respecto Lander (2000) hace un extraordinario análisis sobre el sentido de lo que hacemos en las Universidades venezolanas. Afirma que los saberes de las ciencias sociales y las humanidades juegan un papel medular en el dominio imperial / neocolonial del presente; y que las estructuras homogeneizantes del conocimiento moderno nos hacen presos en el interior de jaulas conceptuales. En un muy bien documentado análisis empírico, demuestra cómo el conocimiento se ha venido utilizando para destruir y dominar. El concepto mismo de desarrollo basado en el consumismo de los llamados países desarrollados, e impuestos por los saberes hegemónicos, lleva hacia la destrucción del planeta.

Aun cuando no está dentro de los objetivos de este trabajo analizar las diferentes concepciones del conocimiento, consideramos necesario señalar las cuatro relaciones que Nicol (1965) dice debe contener el conocimiento científico: la relación epistemológica; la relación lógica (metodológica); la ubicación histórica (contextual) y la relación dialógica. Así como también las etapas y características que señala Bungen (1959) deben cumplirse para que el conocimiento adquiera la categoría de ciencia. Al momento de diseñar un programa de transformación de instituciones que trabajan con el conocimiento, estos aportes es indispensables someterlos a análisis.

Tanto el conocimiento científico como la ciencia, son sistemas abiertos y constituyen los más poderosos instrumentos para el desarrollo del hombre y de la sociedad. Sin embargo como instrumentos que son, pueden ser usados para bien o para mal. Existen en la historia de la humanidad abundantes hechos que demuestran tal afirmación.

Respecto al conocimiento, el legislador venezolano no se pronuncia a favor de ninguna teoría, sin embargo cuando le asigna a la universidades la misión de "buscar la verdad y afianzar los valores trascendentales del hombre", se puede interpretar que subyace una concepción objetivista, ahistórica y cerrada del conocimiento: el conocimiento como "producto" que se puede "enseñar" y no como "proceso" abierto resultante de la interacción HOMBRE $\leftrightarrow$ OBJETO $\leftrightarrow$ HOMBRE.

Concordante con esa interpretación, en el artículo 47 de la mencionada ley se establece:..."Por su especial naturaleza a cada Facultad corresponde enseñar e investigar una rama particular de la ciencia o de la cultura, pero todas se integran en la unidad de la Universidad y deben cumplir los supremos fines de ésta."...También en el artículo 132 referido al Consejo de Desarrollo Científico y Humanístico se establece:..."que tendrá por finalidad estimular y coordinar la investigación en el campo científico y en el dominio de los estudios humanísticos y sociales". En ambos casos hay implícita una concepción disciplinar del conocimiento e incluso una valoración de "científico" a un "campo" del conocimiento y a los demás los denomina "estudios humanísticos y sociales". Parece que los primeros son científicos y los otros no.

Respecto a la concepción del hombre, el artículo 1 de la mencionada Ley de Universidades define a los intereses que reúne a profesores y estudiantes en la tarea de buscar la verdad, como "espirituales", lo cual implica que existen intereses espirituales y materiales y estos no pue- 
den existir sin el sujeto que posea esos intereses, y para tener intereses espirituales hay que tener materia y espíritu. El modelo teórico universitario vigente contiene entonces, una concepción espiritualista del hombre, es decir, considera al hombre como un ser dotado de materia y espiritu.

Aunque hasta hoy no se ha podido demostrar de manera"objetiva", "científica", la existencia del espíritu, tampoco se ha podido demostrar su inexistencia. Pero como hay más fundados indicios y más consenso a lo largo de toda la historia conocida del hombre, de su existencia, es más prudente continuar concibiendo al hombre como un ser dotado de cuerpo y espíritu. El modelo curricular que se proponga debe estar abierto a esta concepción.

Sobre la naturaleza del hombre, Morín (2000: 19) dice: "El ser humano es a la vez físico, biológico, síquico, cultural, social, histórico. Es esta unidad compleja de la naturaleza humana la que está completamente desintegrada en la educación a través de las disciplinas y que imposibilitan aprender lo que significa ser humano".

En cuanto a la educación la ley comentada establece en su artículo 4 "la enseñanza universitaria se inspirará en un definitivo espíritu democrático, de justicia social y de solidaridad humana, y estará abierta a todas las corrientes del pensamiento universal, las cuales se expondrán y analizarán de manera rigurosamente cientifica".

Se desprende de esa lectura que hay que incorporar las más recientes teorías sobre la educación ya que "enseñar" implica sólo mostrar, y en la actualidad se considera a la educación como un proceso de interacción mucho más complejo cuyos múltiples elementos y sus interrelaciones deben ser tomados en cuenta al proponer los cambios necesarios en el modelo teórico universitario. El hecho de que se establezca que "estará abierta a todas las corrientes del pensamiento universal" facilita hacer reformas curriculares que incorporen los nuevos conceptos sobre el conocimiento, la investigación, la educación, la gerencia, etc.

También sobre la educación, Morín (2000) hace una extraordinaria crítica a la concepción educativa que se ha venido aplicando, y al efecto propone lo que él mismo denomina "Los siete saberes necesarios a la educación del futuro": 1. Actitud crítica ante el conocimiento. 2. Los principios de un conocimiento pertinente. 3. Enseñar la condición humana. 4. Enseñar la identidad terrenal. 5. Enfrentar las incertidumbres. 6. Enseñar la compresión. 7. La ética del género humano.

Coincidiendo con Morín (2000) respecto a la concepción de la educación, Mayor, en el prefacio del mencionado libro afirma: "En esta evolución hacia los cambios fundamentales de nuestros estilos de vida y nuestros comportamientos, la educación -en su sentido más ampliojuega un papel preponderante. La educación es "la fuerza del futuro", porque ella constituye uno de los instrumentos más poderosos para realizar los cambios" Morin (2000: 13).

En cuanto al modelo organizativo y de dirección la mencionada ley ubica a las universidades "como parte integrante del sistema educativo, especialmente del área de estudios superiores" (Art. 5); coordinadas por el Consejo Nacional de 
Universidades (art. 18) y aunque gozan de autonomía organizativa (ordinal 1 art. 9) la misma ley les establece una organización por Facultades, Escuelas, Institutos; así como también organismos de dirección y gobierno: Consejo Universitario, Consejo de Facultad, etc. (art. 47 y sgtes.). Tanto la organización como el gobierno parten de una clasificación del conocimiento en disciplinas o campos, que en la actualidad se consideran una seria limitación para su desarrollo y han originado una excesiva burocracia que hace lento los procesos de toma de decisiones, de ejecución, evaluación y control de gestión.

Sólo a título de experimentación debidamente justificada, planificada y sujeta a otros requisitos y límites, se permite a las universidades una estructura académica distinta a la establecida en la ley que comentamos, siempre que ello no comporte alteración en la composición o en la forma de designación o de elección de los órganos directivos de la Universidad (art. 187) (Congreso de la República de Venezuela 1970).

La tendencia actual es a flexibilizar esas clasificaciones, a propiciar la investigación interdisciplinaria y transdisciplinaria y a ampliar los límites de aplicación de los métodos de investigación que servían de base a esas clasificaciones. Así como también, respetando la participación democrática en la elección de los órganos de gobierno, es necesario estudiar la factibilidad de hacer más ágil y eficiente los organismos de dirección.

Se hace indispensable entonces que las universidades venezolanas propongan cambios profundos en sus modelos teóricos y organizativos, en sus dise- ños curriculares, etc. para que puedan estar incorporando permanentemente los más recientes avances de la ciencia. Deben constituirse en instituciones abiertas en permanente proceso de actualización y creación de nuevos conocimientos científicos que les permita cumplir con mayor eficacia, eficiencia, calidad y pertinencia social la misión que la ley le asigna, que continúa teniendo plena vigencia.

\section{Teorías gerenciales para interpretar la gestión universitaria}

Hemos seleccionado algunos aportes de algunas teorías gerenciales que consideramos pueden ser aplicables para la gerencia de la transformación de las Universidades Públicas Autónomas. Sin embargo esto no excluye la posibilidad de aplicar otros aportes teóricos a lo largo de la existencia de estas instituciones, si se logra que sean asumidas como sistema abierto a los avances de la ciencia.

\subsection{Teorías sobre la toma de decisiones}

Como ya expusimos, son las funciones (planificar, dirigir, controlar y evaluar) los elementos que definen la gerencia, y el ejercicio de dichas funciones genera poder. En cualquier organización privada o pública quien o quienes deciden sobre: ¿qué bien o servicio se va a producir?, ¿por qué?, ¿para qué?, cuánto se va a producir?, ¿cómo se va a producir?, ¿con qué se va a producir?, ¿cuándo se va a producir?, ¿dónde?, ¿quién(es) van a producir?, y además poseen los re- 
cursos y medios necesarios para ejecutar lo planificado, son los que detentan el poder y en consecuencia son los que tienen la posibilidad de tomar las decisiones.

La decisión entonces consiste en definir qué hacer, cómo, cuándo, etc., ante alternativas. Implica disponer de información, evaluar las posibles acciones y escoger la que se considere más conveniente.

Pfeffer (2000) analiza la evolución y ejercicio del poder y la influencia. Distingue entre "el ejercicio del control formal, jerárquico" y "el ejercicio de la influencia informal".

El formal tiende a generar resistencia porque implica restricción externa a la libertad individual, en cambio la influencia por liderazgo ejercido en base a cualidades del líder: simpatía, prestigio, confianza, sentimientos, etc. estimula la admiración y genera respuestas positivas sin resistencia.

Sin embargo, el éxito en las organizaciones no puede estar supeditado a que dispongan de un líder o que sus gerentes tengan las cualidades de líder. Es necesario entonces definir el poder, sus elementos constitutivos y la manera como se ejerce para que cualquier trabajo colectivo sea exitoso.

Sobre el poder existen diversas definiciones que contienen también posiciones de sus autores respecto a justificar su uso de manera abierta, autoritaria o de manera encubierta o sutil. Pfeffer (2000) hace un análisis del poder utilizando varias definiciones tomadas de otros autores, a saber:

- "La producción de efectos esperados por algunos hombres en otros hombres" (Rissell, B.)
Señala tres elementos claves en esta definición: 1.La influencia de algunos en otros. 2. El hecho de que el poder no está relegado a las relaciones entre superiores y subordinados. 3 . La idea de que esta influencia es consciente y deliberada.

Establece la distinción entre el poder y autoridad en base a que la autoridad es el poder pero debidamente autorizado y legalizado por la sociedad y en consecuencia es menos arbitraria y notoria y tiene menos resistencia por parte de quienes deben cumplir las decisiones que tomen las autoridades.

- El poder "es la capacidad de controlar a otros" (Wrong, D.)

Aunque es más precisa y específi$\mathrm{ca}$, considero que no hay que identificar el poder con el control, ya que en todo caso el ejercer el poder supone lograr no sólo controlar, sino que los otros hagan lo que se les ha ordenado. El control es un medio para verificar que ha sido hecho lo que se ordenó en el lugar y tiempo previsto.

El uso del poder, o de la influencia interpersonal al igual que cualquier otro instrumento, mecanismo o procedimiento, puede ser usado para bien o para mal, para beneficio de toda la sociedad, de un sector de la sociedad, del Estado o de el individuo y en última instancia depende de los valores éticos y morales de quien o quienes lo detentan.

Cuando el poder se ejerce a las fuerza simplemente se impone a los subordinados y es la forma más eficaz y rápida de obtener resultados a corto plazo, aunque no tome en cuenta que generalmente genera resistencia y rebeldía. Pero cuando se enfrenta a otros poderes en situaciones en las cuales no se tiene 
claro predominio, o cuando conviene aplicar sólo la influencia interpersonal, se debe recurrir entonces a la negociación.

Pfeffer (2000: 211) dice que aunque la mayoría de las definiciones de poder la relacionan con la dominación y la fuerza "preferimos una concepción voluntarista de los actos basada en la elección personal"

En cambio Coriat (1992) plantea que la sustitución, en algunos casos, de la economía de escala de la gran empresa por la especialización flexible, la flexibilidad dinámica y la empresa mediana, no surgió espontáneamente, sino que se da para aumentar el poder sobre los consumidores y obligarlos a comprar con más frecuencia. Tal es el caso de la denominada política de la obsolescencia planificada de la industria automotriz y de otros equipos que antes se consideraban bienes duraderos por su larga vida útil.

El poder se ejerce en el interior de las organizaciones y también sobre los consumidores y proveedores de insumos y servicios, mediante la modificación de las relaciones entre empresas creando las denominadas "asociaciones" y las "redes" (Coriat 1992).

A su vez, Bennett (1997) afirma que la flexibilización de la producción y la constitución de redes de empresas ha permitido la concentración del poder sin centralización, la polarización creciente de los salarios aumentando las desigualdades económicas y sociales y al mismo tiempo, el aprovechamiento de las ventajas locales en diferentes lugares del mundo.

La nueva forma de organización ha trascendido las fronteras de los países y en algunos casos han concentrado sufi- ciente poder como para ejercer influencia en diversos países, por lo que se han realizado esfuerzos en las de $\mathrm{Na}$ ciones Unidas y de otros entes oficiales multinacionales que establecen códigos de conducta y, en el caso de la comunidad económica europea, incluso fijan reglas respecto a las relaciones laborales, al medio ambiente, a la sanidad y a las prácticas sobre seguridad de las corporaciones multinacionales y sus redes de producción (Bennett 1.997: 243-44).

En el caso de las Universidades públicas autónomas de Venezuela que por su naturaleza autónoma y democrática han generado grupos de poder, tiene más relevancia tomar en cuenta los cambios ocurridos en otras organizaciones exitosas respecto a los nuevos mecanismos de control y ejercicio del poder, para diseñar sus respectivos programas de transformación haciendo las adaptaciones correspondientes que los hagan más factibles.

\subsection{Validez de los modelos empresariales para organizar el trabajo universitario}

La aplicación del principio de la racionalidad económica que ha caracterizado la actividad humana relacionada con la producción de bienes y servicios y los intentos por parte de los propietarios del capital de estar permanentemente obteniendo las más elevadas ganancias al menor costo posible, ha dado lugar a estudios cada vez más precisos sobre la división y organización del trabajo, los métodos y técnicas de producción, las formas de organización de las empresas, la 
aplicación de los avances científicos y tecnológicos, para elevar la eficacia, eficiencia, calidad, productividad y rentabilidad.

Aun cuando los más destacados aportes teóricos y experiencias han sido logrados en estudios de las empresas del sector privado, nos proponemos ahora hacer un breve análisis crítico sobre la posibilidad de aplicar algunos de esos aportes a las Universidades Públicas Autónomas de Venezuela.

Aunque la misión de las Universidades Públicas Venezolanas no es obtener ganancias en términos monetarios, sí se puede aplicar el principio de la racionalidad económica puesto que éste implica esfuerzos en varias direcciones: a) Maximizar la ganancia; b) Reducir los costos; c) mejorar la calidad y d) en las tres direcciones.

La eficacia, eficiencia y calidad se puede lograr actuando sobre los recursos en general, sobre los trabajadores, sobre los métodos de división y organización del trabajo, sobre los métodos y técnicas de producción y en general sobre la organización de la empresa y de sus relaciones con el entorno ambiental y social.

El aprovechamiento eficaz y eficiente de los recursos sin destruirlos, con adecuados métodos y técnicas de conservación sin afectar el ambiente debe ser objetivo común de toda organización.

Taylor (1983) y Fayol (1983) considerados los creadores de la denominada administración científica o escuela tradicional enfocaron sus rigurosos estudios científicos de la empresa privada hacia la división y organización del trabajo en el interior de las empresas.
Taylor (1983) en su obra Principios de la Administración Científica acertadamente afirma: "El objeto principal de la administración ha de ser asegurar la máxima prosperidad para el patrón, junto con la máxima prosperidad para cada uno de los empleados" (Taylor 1983: 19).

Con esta afirmación refuta la creencia muy generalizada de que los intereses del patrón y los trabajadores son antagónicos. Si la prosperidad del patrón conlleva también la prosperidad de los trabajadores, porque éstos reciban mejores remuneraciones y condiciones más favorables de trabajo, la prosperidad deberá ser un objetivo común a patronos y trabajadores, pero no todos los patronos aceptan este principio ni mucho menos lo aplican. Entonces sí, el trabajador actuando de acuerdo al principio de la racionalidad económica, trata de hacer el menor esfuerzo posible porque su ganancia (la remuneración) es constante y no está en función del esfuerzo.

La tendencia a trabajar lo menos posible se origina, según Taylor (1983) por tres causas: 1 . La "mentira" de que si los trabajadores aumentan el rendimiento ocasionará reducción de personal. 2. Los defectuosos sistemas de administración que no controlan adecuadamente y premian el rendimiento de cada trabajador. El bajo rendimiento a su vez se origina por dos causas: 2.1. Poco rendimiento natural: hay personas lentas y personas rápidas. 2.2. Bajo rendimiento sistemático. Algunos patronos pretenden ahorrarse dinero si pagan por día y no "a destajo" o "por pieza". 3. Los ineficientes métodos establecidos "a ojo de buen cubero" sin 
hacer estudios del proceso, la secuencia, el tiempo, etc.

Hace la salvedad de que hay que tomar en cuenta las particularidades de cada empresa ya que "la prosperidad depende de tantos factores que quedan completamente fuera de control de cualquier conjunto de hombres o de cualquier estado o hasta de cualquier país, que forzosamente han de surgir ciertos períodos en los que ambas partes habrán de sufrir" (Taylor 1983: 34).

Hay que sustituir la administración empírica de iniciativa y estímulo, por la administración científica y al efecto propone los principios de la administración científica, a saber: 1.El establecimiento de una verdadera ciencia (estudio de cada caso particular de organización). 2 . La selección científica del trabajador. 3. Su educación y formación científica. 4. La colaboración estrecha y amistosa entre la dirección y los trabajadores.

En el caso de las universidades públicas la prosperidad del patrón, que en este caso es el Estado Venezolano, es también la prosperidad del trabajador puesto que forma parte de la nación venezolana que representa el Estado y podrá el mismo disponer de educación de calidad y gratis, de los aportes de los investigadores y de todos los servicios que prestan estas instituciones. Pero como estos beneficios no son muy inmediatos y evidentes, habrá que hacer los estudios científicos correspondientes a cada caso para organizar el trabajo y en base a los principios del Taylorismo que sean aplicables, establecer un sistema de selección científica del personal y de remuneración en base al rendimiento in- dividual, así como la actualización y formación permanente de sus integrantes.

En el caso de Fayol es importante destacar que amplía la concepción de la administración y el ámbito de su aplicación. Al efecto expresa: "Administrar, es prever, organizar, dirigir, coordinar y controlar" (Fayol 1.983: 138) y es sólo una de las 6 operaciones que se realizan en las empresas: técnicas, comerciales, financieras, de seguridad, de contabilidad y administrativas.

"Mi doctrina administrativa, tiene por objeto facilitar el gobierno de empresas, sean industriales, sean militares o de cualquier índole. Sus principios, sus reglas y sus procedimiento deben, pues, responder tanto a las necesidades del Ejército como a las necesidades de la industria" (Fayol 1.983: 132) (destacado nuestro).

Sin embargo advierte: "No hay nada rígido ni absoluto en materia administrativa; todo en ello es cuestión de medida. No hay que aplicar casi nunca dos veces el mismo principio en condiciones idénticas; es preciso tener en cuenta circunstancias diversas y cambiantes, hombres igualmente diversos y cambiantes y muchos otros elementos variables" (Fayol 1.983: 157).

Los principios de la administración que, él mismo señala, ha tenido ocasión de aplicar con más frecuencia, son: 1.La división del trabajo. 2. La autoridad. 3. La disciplina. 4. La unidad de mando. 5. La unidad de dirección. 6. La subordinación de los intereses particulares al interés general. 7. La remuneración. 8. La centralización. 9. La jerarquía. 10. El orden. 11. La equidad. 12. La estabilidad del per- 
sonal. 13. La iniciativa. 14. La unión del personal.

Dentro del programa de transformación hacia el interior, que deben diseñar las universidades públicas autónomas de Venezuela se debe tomar en cuenta estos principios y estudiar la factibilidad de su aplicación en cada caso particular.

En la evolución de los estudios sobre la administración, entendida como ya se expuso, por las funciones de planificar, dirigir, controlar y evaluar, surgieron nuevos aportes que analizan los problemas de la gerencia desde una perspectiva más amplia aún, puesto que consideran al hombre no sólo en el contexto de la empresa y en forma individual, sino en el contexto global social, sociológico, cultural, etc.

Surge así la denominada Escuela de las Relaciones Humanas que se caracteriza "por plantear como punto de partida una concepción del hombre opuesta a la del tradicionalismo, y por proponer, como consecuencia práctica, departamentos de personal con importantes diferencias en su naturaleza y funciones, respecto a los tradicionales" (Kliksberg 1975: 56).

Según la concepción del hombre de la Escuela de las Relaciones Humanas el hombre no sólo actúa en forma individual mediante el estímulo del premio y el castigo, sino que por su instinto gregario actúa también por influencia del grupo al que pertenece y con el cual se identifica. Esto les permite señalar otras fuentes de autoridad en las organizaciones. Consideran que junto al líder forzoso o jefe impuesto por la empresa, coexisten el líder tecnológico, el líder político, el líder centralizador de comunicaciones, el líder sociológico que dispone también de autoridad y poder efectivo.

El hombre pertenece a su grupo de trabajo, a grupos informarles, a grupos de presión, a grupos extraempresa de pertenencia y referencia, al sindicato, etc. Este hecho determina que los llamados y los planes que no tengan en cuenta la influencia sobre su conducta de los factores grupales y sociales están condenados al fracaso ...El "grupo humano" es una realidad actuante, y enormemente influyente sobre el individuo (Kliksberg 1975: 7).

La importante influencia que ejercen los diferentes grupos de los cuales forma parte el trabajador, quedó además demostrada con los experimentos realizados en los talleres Hawthorne de la Western Electric Co. de Chicago en 1924 , entre cuyos aportes se pueden citar: 1. Grado de satisfacción en las relaciones internas en el grupo. 2. Grado de satisfacción en las relaciones con el supervisor. Es mejor el sistema de supervisión descentralizado y de control de resultados, que el centralizado y sobre cada paso o tarea. 3. Grado de participación en las decisiones. Es mejor el liderazgo participativo que el legalista o impuesto. 4. Grado de información sobre el trabajo y sus fines. 5. Descubrimiento de la existencia en la empresa de numerosos grupos informales, estructurados socialmente, con liderazgo y normas propias (Kliksberg, 1975).

El cumplimiento de las órdenes (la subordinación de los trabajadores) no es "mecánico" sino que depende de múltiples circunstancias. La sumisión podrá llegar a ser total pero no por el premio y el 
castigo sino por una "delegación de abajo hacia arriba" producto de una alta identificación con los objetivos de la organización y de permitir, mediante estrategias de negociación, la participación de grupos y de líderes que tengan poder sobre los trabajadores.

En el caso de las Universidades y demás organismos públicos el proceso de selección, formación y entrenamiento del personal debe estar dirigido a lograr una alta identificación con los objetivos de la institución y una elevada dosis de vocación de servicio, solidaridad, honestidad, responsabilidad, disciplina, etc., a fin de poder lograr las metas de las Relaciones Humanas a saber: "1. Una institución en estado de armonía. 2. Sin conflictos. 3. Con espíritu de cuerpo. 4. Con alta identificación del personal con los objetivos de la institución" (Kliksberg, 1975: 92).

Cualquier programa de transformación en las universidades públicas autónomas de Venezuela debe incluir un proyecto específico y factible que además de los premios y castigos, tenga en cuenta la influencia de los grupos sobre los trabajadores y produzca cambios de conducta de tal manera que todo el personal forme una verdadera comunidad y hagan suyo los objetivos de cada institución.

Los cambios más recientes en las empresas y en las correspondientes teorías para explicarlos, han estado relacionados con los avances científicos y tecnológicos aplicados a la producción y enfocados hacia las relaciones con el entorno: el mercado, los proveedores, el Estado, la flexibilización de la producción, la calidad total, las asociaciones estratégicas, las redes de producción, etc.
También las universidades deben flexibilizar la producción de sus servicios de docencia, investigación y extensión, ya que deben asimilar los cambios producidos en el entorno para poder analizarlos, proponer los nuevos ajustes y cambios que sean necesarios y ponerlos al servicio del desarrollo de nuestra nación. Esta nueva misión constituye un cambio en la "Teoría de la Empresa" que como lo expresa Drucker: "los supuestos sobre los que se ha construido y se gobierna la organización ya no encajan en la realidad" (Drucker (2002: 60) También las asociaciones estratégicas y la constitución de redes con productores y usuarios del conocimiento deben incorporarse como posibles estrategias de elevación de los niveles de eficacia, calidad, eficiencia y pertinencia social.

Las universidades públicas autónomas de Venezuela deben, por mandato de su respectiva Ley, no sólo crear y desarrollar el conocimiento sino también asimilarlo, difundirlo y aplicarlo al servicio del desarrollo de la nación, en consecuencia uno de los más importantes proyectos dentro de un programa integral de transformación, debe ser mantenerse actualizada en todos los avances científicos y tecnológicos y evaluar las posibilidades de aplicación en nuestra nación.

Aun cuando existen propuestas de transformación enfocadas a poner a las Universidades en sintonía con los avances científicos y tecnológicos, entre las cuales podemos mencionar la de Méndez, Evaristo "Hologerencia Académica" (2004) y la de la Asociación Venezolana de Rectores Universitarios (AVERU) (2001), hasta ahora no se ha logrado ponerlas en marcha. 


\subsection{El control empresarial y el control universitario}

Uno de los principales problemas que han enfrentado quienes gerencian procesos productivos a lo largo de la historia de la humanidad, es lograr la coordinación, eficacia, calidad y eficiencia del trabajo humano colectivo. El éxito de cualquier organización depende en gran medida de que sus directores logren que los trabajadores hagan las cosas bien, rápido y con el menor esfuerzo y desperdicio posible. Esto exige mecanismos de control y evaluación eficaces y eficientes que además de posibilitar la consecución de los objetivos de la organización, faciliten el ejercicio del poder de los dirigentes sobre los dirigidos.

Según Gamboa y Suárez (1977: 56):

El término control sintetiza diferentes medios utilizados por quien ejerce el dominio sobre otros sujetos, para asegurar a lo largo de un proceso, el logro de los objetivos o metas previstos [...].

El control se clasifica en ideológico y represivo o coercitivo [...]. El represivo "es aquel que utiliza la fuerza, la amenaza o cualquier medio coercitivo para evitar las alteraciones a la norma de comportamiento establecida y para represar manifestaciones de descontento o rebeldía por desacuerdo con la norma [...] utiliza directa o indirectamente diversos tipos y grados de violencia contra los sujetos controlados: violencia física, presión y diversos tipos de sanciones o formas más sutiles, como la amenaza. Una forma de control coercitivo es el control técnico.
El control ideológico es más sutil, es aquel que logra ciertos comportamientos de los individuos inculcando determinadas ideas por medio de la persuasión, la inducción, la manipulación o la concesión de algunos beneficios materiales que legitimen a quienes detentan el poder. Los fundamentos del control ideológico se encuentran en los sistemas religiosos, de parentesco y jerárquico ceremonial, que legitiman las relaciones de autoridad; se reafirman en el sistema escolar que establece mecanismos rigurosos de selección-eliminación y de jerarquización con base en el saber (destacado nuestro). En las empresas privadas el elemento más predominante en el control sobre la eficacia, calidad, eficiencia se ejerce a través de la propiedad privada de los recursos y medios de producción. Los propietarios por si mismos a través de sus gerentes ejercen el poder mediante los mecanismos de premio y castigo. Los trabajadores cumplen sus funciones bajo la presión sicológica y real, de que si no logran las metas impuestas por el patrón, pueden ser despedidos. Aún cuando es uno de los más eficaces mecanismos de control, la empresa privada también usa los demás mecanismos de control, ideológicos, de relaciones humanas, legales, etc.

En el sector público, aun cuando el Estado pudiera considerarse de manera similar al patrono del sector privado y de hecho, legalmente es el patrono, hay que tomar en cuenta algunos elementos que disminuyen la eficacia del mecanismo de control a través de la propiedad de los medios de producción. 
Los propietarios de los bienes públicos son todos los ciudadanos, los Gerentes o Directores de los organismos públicos así como sus trabajadores no tienen igual estímulo al que se tiene en la empresa privada cuando se establece la remuneración de los trabajadores en base al rendimiento individual. Este es un mecanismo de control que debe ser objeto de un proyecto específico para estudiar la factibilidad de su aplicación en las Universidades. A pesar de las críticas respecto a que la división del trabajo en tareas y el pago a destajo, aumenta la tasa de explotación del trabajador, en el caso del sector público, el incremento de la productividad que puede generar en términos de eficacia, calidad y eficiencia de los servicios públicos que presta, beneficiaran a la sociedad en general y no a un empresario en particular. El principio de la racionalidad económica es lo que garantiza eficiencia en el proceso de producción de bienes y servicios independientemente del sector en el cual se aplique; el problema de la explotación radica en el uso que se le dé a el excedente del valor generado, resultará injusto si el beneficio es sólo para el propietario de los medios de producción, pero si el beneficio es por igual para todos los ciudadanos de una nación, resultará justa y necesaria su aplicación.

Otro de los elementos que disminuye la eficacia de los mecanismos de control y la aplicación del principio de la racionalidad económica en el sector público es, para fraseando a Halloway (1982: 52), que el Estado no cuenta con un grado suficiente de unidad, sino que es en esencia "un conglomerado de unidades centradas en si misma", cada una persiguiendo sus propios intereses particulares y de grupos que obtienen poder y luchan por conservarlo y acrecentarlo, lo cual disminuye las posibilidades de una eficaz coordinación y control de gestión. En el caso de las Universidades Públicas Autónomas de Venezuela, diversos diagnósticos coinciden en que son un conglomerado de unidades centradas en sí misma (Facultades, Escuelas etc.) cada una persiguiendo sus propios intereses particulares y de grupos, que obtienen cuotas de poder por el sistema de gobierno establecido en la Ley de Universidades. Se plantea, entonces, la necesidad de que cualquier proyecto de transformación en el que se pretenda elevar la eficacia, calidad y eficiencia debe ser abordado mediante estrategias de planificación participativa y negociación con los grupos que tengan poder dentro de las mismas unidades.

En las universidades el objeto de trabajo es el conocimiento y los miembros de su respectiva comunidad (profesores y estudiantes); $y$ en las complejas organizaciones sociales de la actualidad, el conocimiento es quizás el elemento más importante para alcanzar el éxito en la búsqueda de soluciones a los problemas relacionados con el desarrollo de la nación.

Es indispensable entonces que cualquier programa de reforma tenga en cuenta, entre otras cosas, los seis (6) factores que Drucker (1999) señala como determinantes de la productividad de los que trabajan con el conocimiento: 1. Definición precisa de la tarea. Concepción del conocimiento, de la ciencia, sus métodos, etc., del programa y del proyecto específico, etc. 2. La autodirección o autonomía del trabajador. 3. La innovación 
permanente. 4. El aprendizaje y enseñanza permanente. 5. En el control de la productividad debe dársele igual importancia a la calidad que a la cantidad. 6 . EI trabajador intelectual debe ser tratado como un activo de la empresa y no como un gasto. Recibir las mejores remuneraciones y beneficios sociales y debe desear trabajar para la institución por encima de otras. Es decir, estar identificado con ella.

Establecer un adecuado sistema de control en el que predomine el control ideológico y de resultados, sobre el represivo, las técnicas de planificación participativa, las estrategias de negociación tanto al interior de las instituciones como en sus relaciones con el entorno, debe ser un proyecto prioritario dentro de un programa de transformación de las universidades, para poder elevar permanentemente sus niveles de eficacia, calidad, eficiencia y pertinencia social.

\subsection{Validez de los criterios de gestión para evaluar las universidades}

"Evaluar implica en principio estimar el "valor" cualitativo o cuantitativo de un resultado obtenido o posible" (Gamboa y Suárez 1997: 57) Implica contrastar lo realizado con lo planificado para hacer los ajustes que resulten necesarios. Comparar lo que es con lo que debe ser, para valorarlo y mejorarlo. La estimación del "valor" requiere que el evaluador tenga un modelo previamente definido, un patrón de comparación o referente comparativo.

En el caso de la empresa privada, aun cuando cada empresa puede definir su propio modelo de organización, el objetivo principal y común es obtener permanentemente la máxima ganancia al menor costo posible. En consecuencia los criterios de evaluación e indicadores aplicables son los relacionados con la eficacia y productividad y son impuestos por los propietarios.

En el caso de las universidades venezolanas, el referente comparativo se encuentra contenido en la Ley de Universidades vigente, que establece su definición, misión, objetivos, recursos, organización, gobierno, etc.

Sin embargo en estos momentos en que la constitución de la República Bolivariana de Venezuela aprobada en 1999 define a la educación junto con el trabajo como instrumento para el desarrollo personal y colectivo (Asamblea Nacional Constituyente 1999: art. 3) y que además se encuentran en estudio en la Asamblea Nacional un nuevo proyecto de Ley de Educación y otro de Ley de Educación Superior, es el momento oportuno para que sean las propias universidades las que propongan un nuevo modelo de Universidad en la cual se asuma como misión fundamental ser instrumento para el permanente desarrollo de la nación. Ese nuevo modelo de universidad seria el nuevo referente comparativo que debería establecer mecanismos que permitan la apertura a todos los avances de la ciencia.

Los criterios para la evaluación deben ser de acuerdo con los objetivos previamente definidos, que a su vez deben formar parte del modelo teórico o referente comparativo de cada universidad y ser expresado con suficiente precisión como para permitir el establecimiento de crite- 
rios de evaluación y los correspondientes indicadores.

El mismo criterio de la racionalidad económica que se aplica en la empresa privada, puede ser aplicado también a las universidades, pero con la variante de que el indicador debe ser la población servida con la máxima calidad y la máxima cobertura de la demanda de los servicios que presta. Para cada una de las funciones que la Ley le asigna a las universidades: Docencia, Investigación y Extensión deben definirse programas y proyectos en los cuales se expresan los objetivos generales y específicos, que por tratarse de servicios públicos deben ser evaluados en base a gestión, resultado expresado en términos de estudiantes inscritos y atendidos en relación a la demanda, estudiantes graduados, tiempo de permanencia para la graduación, etc. Trabajos de investigación, resultados de la investigación, trascendencia de la investigación, etc. y respecto a la extensión, la vinculación de la investigación y docencia con las demás empresas e instituciones del entorno, trascendencia de los servicios prestados, etc. En general el control de gestión, la evaluación de resultados y las auditorias externas pueden ser los mecanismos de control más apropiado para estas instituciones.

La evaluación implica también un diagnóstico que permita detectar fallas, determinar causas, formular juicios y elaborar propuestas para superarla. La evaluación en las organizaciones forma parte indispensable de cualquier mecanismo de control, ya que al "estimar el valor" de algo o de alguien, se está juzgando la calidad de algo, los resultados de un proce- so, el trabajo individual o colectivo, su eficacia y/o eficiencia, etc.

Es pues indispensable, en todo trabajo humano colectivo, que sus integrantes sepan que existen mecanismos mediante los cuales va a ser evaluado su esfuerzo, su aporte en pro de los objetivos generales y específicos de la organización para que exista un estímulo adicional (premio o castigo) que fortalezca la voluntad del trabajador para cumplir su tarea de la manera más eficaz y eficiente posible.

El control y evaluación influyen de manera preponderante en los niveles de calidad, eficacia y eficiencia del trabajo humano. Sin embargo existe, también en este aspecto, fundados indicios que nos hacen dudar que se hayan logrado los más elevados niveles de calidad, eficacia y eficiencia posibles, en los servicios que prestan las Universidades Públicas Autónomas de Venezuela. Un proyecto específico dentro del programa de transformación, será necesario para hacer un diagnóstico que permita detectar fallas, determinar causas y hacer propuestas para mejorar los sistemas de evaluación y control.

En el caso de la empresa privada el ejercicio del poder mediante el control coercitivo, permite a los propietarios tomar las decisiones para estar permanentemente haciendo los cambios necesarios para ajustarse al mercado y/ ó resolver los problemas que se presenten en su organización.

En el caso de las Universidades Autónomas Venezolanas, haciendo uso de su autonomía pueden diseñar, planificar y ejecutar su propio y particular proceso de transformación (artículos 9 y 187 de 
la Ley de Universidades) en el cual establezcan, entre otras cosas, los criterios de evaluación, los indicadores y demás componentes y requisitos que deben conformar el respectivo programa de transformación.

Sin embargo, dada las características de " conglomerados de unidades centradas en sí mismos" y a las cuotas y redes de poder que detentan grupos de electores dentro de ellas, a los cuales nos referimos antes, será necesario usar técnicas de planificación participativa y estrategias de negociación para construirle viabilidad al proceso.

\subsection{Relaciones entre las universidades y el Estado}

La ley de Universidades les delimita su autonomía y establece claramente la subordinación de todas las Universidades a el Estado y al efecto, en el Titulo II, se crea el CONSEJO NACIONAL DE UNIVERSIDADES que es el organismo encargado de ... "coordinar las relaciones de ellas entre sí y con el resto del sistema educativo, de armonizar sus planes docentes, culturales y científicos y de planificar su desarrollo de acuerdo con las necesidades del país..." (Congreso de la República de Venezuela, 1979: art. 18). Lo preside el Ministro de Educación quien forma parte del poder ejecutivo o gobierno nacional, que esté en determinado momento representando y gerenciando al país.

Los servicios de docencia, investigación y extensión que ellas prestan deben ser para el desarrollo de la nación. Aunque no existe consenso sobre el concepto de desarrollo, a los fines de este análisis y para mantener abierta la posibilidad de que se incorporen los nuevos aportes teóricos que puedan surgir, se propone una concepción del desarrollo también abierta y flexible: "Proceso mediante el cual el hombre, socialmente considerado, dirige su constante esfuerzo hacia un conjunto de condiciones ideales (humanas y ambientales) que definen su bienestar y le permiten la permanente superación para sí y sus semejantes" (Romero, 1988: 18). En todo caso tampoco hay dudas respecto al uso que los trabajadores del conocimiento deben darle a los servicios que prestan.

Para alcanzar el desarrollo de la nación, si bien es cierto que el conocimiento científico, la educación y la técnica son instrumentos indispensables, también son necesarios los recursos naturales, el trabajo, el capital, la organización social, los servicios públicos, las leyes, las vías, redes y medios de comunicación y especialmente el Estado con su función fundamental de ser el coordinador de las actividades del grupo humano que conforma la nación. Por razones de funcionalidad, y de acuerdo con los principios de unidad de dirección y unidad de mando señalados por Fayol (1983) sólo puede haber un único ente que coordine las actividades de una nación.

Las relaciones entonces, deben ser de mutua colaboración, de servicio recíproco y no de antagonismo como lamentablemente ha ocurrido, en algunos casos, en Venezuela.

Independientemente de las posiciones ideológicas, de quienes dirijan el Estado y las Universidades, la comunidad científica, los universitarios, están en la obligación moral y legal de ofrecer sus 
conocimientos a toda la nación, sin pretender imponer sus puntos de vista e ideología. Por su parte el Estado está obligado por la mencionada Ley a respetar su autonomía, a proporcionar los recursos y a ejercer el control y evaluación.

Sobre este aspecto también es necesario y urgente que en el programa de transformación se incluya un proyecto específico para disminuir la conflictividad y armonizar las relaciones entre las Universidades y el Estado.

Al interior de las Universidades, el gobierno universitario y el de cada Facultad, Escuela, etc., por mandato de la ley, debe ser elegido por votación (régimen democrático) y esto ha originado redes de poder que en algunos casos han resultado serias limitaciones a los cambios. Para superar estas resistencias, será necesario diseñar estrategias muy bien elaboradas y negociadas con los actores para que le den viabilidad política.

\section{Conclusiones y Recomendación}

- Es indispensable y urgente que las Universidades Públicas Autónomas realicen ellas misma un proceso de transformación integral que, tomando en cuenta las nuevas teorías del conocimiento en general y del área de la gerencia en particular, les permita continuar y mejorar el desempeño de su papel de ser "rectoras de la educación, la cultura y la ciencia" y asumir la nueva misión de ser instrumento de desarrollo personal y colectivo que les asigna la Constitución Nacional.
- Los aportes de las teorías utilizados en este trabajo para analizar el modelo universitario contenido en la Ley de Universidades, nos permitieron detectar fallas en áreas muy importantes relacionadas con el poder, la toma de decisiones, el control, la evaluación, la organización del trabajo, etc.

- Los resultados del análisis crítico tanto de las teorias gerenciales utilizadas como del modelo universitario, nos permitieron hacer algunas reflexiones y una propuesta que esperamos sea útil para facilitar los cambios.

- Corresponde a los propios organismos gerenciales de las Universidades Públicas Autónomas, diseñar, promover, dirigir y controlar el proceso de transformación, y en consecuencia en el respectivo programa deben establecer estrategias para que dichos organismos tomen las decisiones necesarias.

- Un proceso de transformación en instituciones como las Universidades que constituyen "un conglomerado de unidades centradas en sí misma" y en las cuales existen grupos formales e informales y redes de poder, sólo es posible mediante técnicas de planificación participativa y estrategias de negociación que permitan formular un programa flexible y negociado, que sirva de marco para que los participantes tengan oportunidad de expresar sus expectativas, proponer ideas, proyectos, etc. y darle viabilidad al proceso.

La recomendación es a las autoridades de cada Universidad para que analicen la idea de convocar a los represen- 
tante de todos los grupos -sin excluir a los opositores- para negociar un programa de transformación abierto a la participación de toda la comunidad universitaria a fin de iniciar un proceso de diagnóstico, recolección de expectativas, ideas, proyectos y sobre todo, lograr el compromiso de parte de los grupos y redes de poder para formular, con las técnicas de planificación participativa, un programa general flexible con proyectos específicos en los cuáles se decida: ¿qué se va a hacer?, ¿porqué?, ¿para qué?, ¿quién(es) lo van a hacer?, ¿con qué?, etc., así como también diseñar programas de motivación y entrenamiento a el personal que tendrá a su cargo la puesta en marcha del proceso de transformación.

\section{Referencias Bibliográficas}

Asamblea Nacional Constituyente (1999). Constitución de la República Bolivariana de Venezuela. Gaceta Oficial № 36860. Venezuela.

Asociación Venezolana de Rectores Universitarios (AVERU) (2001). Estrategias de cambio para la educación superior venezolana. wwwgoogle.com. consultado el 25-04-2007.

Bennett, Harrison (1997). La empresa que viene. La evolución del poder empresarial en la era de la flexibilidad. España. Ediciones Paidós, S.A.

Bungen, Mario (1959). La ciencia su método y filosofía. Argentina. Universidad de Buenos Aires.

Congreso de la República de Venezuela (1970) Ley de Universidades. Gaceta Oficial № 1.429 extraordinaria. Venezuela.

Coriat, Benjamín (1992). El taller y el Robot: Ensayo sobre el Fordismo y la produc- ción en masa en la era de la electrónica. México. Siglo XXI Editores.

Drucker, Peter (1992). La gerencia. Tareas, responsabilidades y prácticas. Argentina. El Ateneo.

Drucker, Peter (1999). Los desafíos de la gerencia para el siglo XXI. Colombia. Grupo Editorial Norma.

Drucker, Peter (2002). Escritos fundamentales. Tomo 2. El Management. Buenos Aires, Argentina. Editorial Sudamericana.

Fayol, Henry (1983). Administración industrial y general. México. Herrero Hnos.

Gamboa C. Teresa y Suárez, Cira (1997) Control y evaluación: funciones claves del Poder económico. Revista Venezolana de Ciencias Sociales. Vol. 1 № 1. pp. 53-75.

Holloway, John (1982). Fundamentos teóricos para una crítica Marxista de la Administración Pública. México. Ediciones INAP.

Ibarra, Eduardo (1993). La Universidad ante el espejo de la excelencia. Mexico. Universidad Autónoma Metropolitana.

Ibarra, Eduardo (1996). Educación superior y teoría de la organización: posibilidades y problemas de una relación impostergable. Revista Universidad Futura Vol. 7 № 20-21.

Kliksberg, Bernardo (1975). El pensamiento organizativo: del Taylorismo a la teoría de la organización. Estrategias modernas en administración: Relaciones humanas y teoría de la organización. Argentina. Editorial Paidós.

Lander, Edgardo (2000). ¿Conocimiento para qué?, ¿Conocimiento para quién? Reflexiones sobre la geopolítica de los saberes hegemónicos. Revista Venezolana de Economía y Ciencias Sociales. Caracas, Venezuela. FACES-UCV. Vol.6 № 2. pp. 53-72. 
Méndez, Evaristo (2004). Hologerencia Académica. Maracaibo, Venezuela. Ediluz.

Morin, Edgar (1997). Introducción al pensamiento complejo. Barcelona, España. Editorial Gedisa, S.A.

Morin, Edgar (2000). Los siete saberes necesarios a la educación del futuro. Caracas, Venezuela. Ediciones FACES-UCV.

Muro, Xiomara (2004). La Gerencia Universitaria. Caracas, Venezuela. Ediciones Opsu.

Nicol, Eduardo (1965). Los principios de la ciencia. México. Fondo de Cultura Económica.
Ochoa, Haydee (1995). Tecnocracia y empresa pública en Venezuela. 19481991. Maracibo, Venezuela. Ediluz.

Pfeffer, Jeffrey (2000). Nuevos rumbos en la teoría de la organización. Problemas y posibilidades. New York, USA. Oxford University Press.

Romero, Néstor (1988). Reflexiones para una nueva concepción del desarrollo y del subdesarrollo. Maracaibo, Venezuela. Ediluz.

Taylor, Frederick (1983). Principios de la Administración Científica. México. Herrero Hnos.

Yarzábal, Luis (1999). Consenso para el cambio en la educación superior. Caracas, Venezuela. IESALC-UNESCO. 